\title{
We Hommage à Jacqueline Barus-Michel
}

\begin{abstract}
J acqueline Barus-Michel nous a quittés le 17 avril 2015. Elle venait de « monter en scène » pour y présenter un Impromptu dans le cadre d'un colloque organisé par le RISC, le tout récent réseau international de sociologie clinique, quand un malaise l'a terrassée. Elle est décédée quelques jours après à l'hôpital.

Cette disparition brutale, à la Molière, nous touche profondément, comme tous ceux qui ont travaillé longtemps avec elle, tous ceux, étudiants, enseignants, chercheurs, praticiens qui ont connu la force de ses engagements, sa passion épistémophilique, son goût du verbe et de la scène, son regard malicieux, son irréductible indépendance, sa vitalité, sa générosité et son autorité.

Jacqueline a jusqu'au bout témoigné de cette "pulsion sociale ", celle qu'elle a cherché à théoriser comme visant les autres, en quête de ce besoin incoercible d'être au milieu de ses semblables, aimée et reconnue par eux. Dans l'ouvrage réalisé en son hommage ${ }^{[1]}$, suite à un colloque organisé en 2009 par le Laboratoire de Changement Social sur son ouvre, elle introduit sa propre intervention ainsi : “ J'ai toujours dit (que Freud me pardonne !) que la reconnaissance qui mêle le psychique et le social était aussi fondamentale que la sexualité : elle fait exister, c'est une seconde naissance au sein des autres, pendu, nourri à la mamelle sociale. Être reconnu par ceux qu'on aime, qu'on admire, qui comptent et sur lesquels on compte, les socii, les compagnons, et même les rivaux, est justement, selon le titre de ce colloque, une expérience subjective en situation sociale!» (2009, p. 257). Cette pulsion sociale, Jacqueline la définit encore comme profondément ambivalente, mêlant nécessité à faire et à être avec les autres et intolérance aux autres. L'irréductible conflictualité qui hante le lien social n'est pas damnation car, écrit-elle " le conflit n'est pas une maladie dont on guérit, c'est la condition de la vie en société, de la relation aux autres et de la vie psychique » (2009, p.264).

Nous retracerons d'abord brièvement son parcours avant de présenter quelques axes centraux de son ouvre.

J. Barus-Michel a d'abord exercé à Tours, comme psychologue et psychothérapeute, dans différentes institutions comme les services de psychiatrie du CHU

[1] Giust-Desprairies Fl., de Gaulejac V. (2009). La subjectivité à l'épreuve du social, Hommage à Jacqueline Barus-Michel, Paris, L'Harmattan.
\end{abstract}


d'Indre-et-Loire, dans des centres de sauvegarde de l'enfance, mais aussi comme expert auprès du Tribunal pour enfants et aux Assises, tout en assurant des charges de cours en psychopathologie à l'Université de Tours.

En 1968, elle poursuit ses activités d'enseignement en psychologie sociale et psychologie clinique à l'université Paris VII, puis elle est recrutée comme assistante. Elle réalise et soutient une thèse de $3^{\mathrm{e}}$ cycle en 1970, intitulée Esquisse d'une phénoménologie sociale de la folie, sous la direction de Jean Duvignaud. Elle accédera à la fonction de professeure des universités, en 1983, après la soutenance de sa thèse d'État, Théorie de la pratique en psychologie sociale clinique.

Elle créera au sein de l'UFR de Sciences Humaines Cliniques une fillière d'enseignement intitulée “ Pratiques, Institutions et Organisations » qui réunissait un grand nombre d'étudiants engagés dans le dispositif clinique proposé au cour duquel s'inscrivait l'analyse des pratiques. La pédagogie y était pensée comme processus d'analyse permettant la mise au travail de l'implication et le dégagement du sens de l'expérience de chacun. Puis, elle créera un DESS intitulé “Intervention dans les organisations ", en collaboration avec A. Lévy et en association avec l'Université Paris 13.

Jacqueline Barus-Michel s’inscrit, à partir de 1988, dans la lignée de ces femmes de renom qui ont créé puis dirigé le Laboratoire de psychologie clinique individuelle et sociale de Paris VII : Juliette Favez-Boutonnier d'abord (jusqu'en 1974) puis Claude Revault d'Allonnes. L'UFR de Sciences Humaines Cliniques de Paris 7 s'orientant progressivement vers le seul référentiel psychanalytique ${ }^{2]}$, elle rejoindra alors l'UFR de Sciences Sociales de cette même université et le Laboratoire de Changement Social dirigé par Vincent de Gaulejac.

Membre fondateur du Centre International de Recherche Formation et Intervention Psychosociologique (crée en 1993), elle sera aussi rédactrice en chef, avec Gilles Amado, de la Nouvelle Revue de Psychosociologie ${ }^{[3]}$, créée en 2006.

Elle a aussi, tout au long de ces années, réalisé un grand nombre d'interventions, de formations, d'animations de groupes d'analyse des pratiques professionnelles, dans des organisations très diverses : attachée au "terrain ", elle a développé son élaboration théorique " au risque de la clinique ». Elle signifie ainsi cette impossibilité d'indiquer un idéal à poursuivre, un lieu où conduire les collectifs, des modèles que la théorie aurait dessinés ou même éclairés. "Ce qui condamne à se tenir en position d'assistant dans une démarche qui demeure celle du client et qui le laisse dans des démêlés renouvelés, des conflits et des contradictions internes et externes. L'intervention ne peut pas être un traitement» (2009, p. 266).

Compagne irréductible de l'instituant, Jacqueline Barus-Michel a, tout au long de sa vie, cherché à construire cette psychologie sociale clinique dont elle est la fondatrice

[2] Cette UFR a changé de nom depuis : elle est devenue « UFR Études Psychanalytiques ».

[3] La Nouvelle Revue de Psychosociologie remplace en 2006, suite à un changement d'éditeur, la Revue Internationale de Psychosociologie, née en 1994 et à laquelle Jacqueline participait activement. 
et à mettre ses analyses à l'épreuve. “ C'est le déblocage des situations et ces mobilités retrouvées qui répondent au terme d'épreuve : l'analyse est éprouvée comme déclencheur de mouvement dans le sens d'un gain, d'une avancée vers un but qui ne peut être défini ou, s'il l'est, seulement par les sujets comme plus larges potentialités » (1996, p. 280).

\section{WE La psychologie sociale clinique}

“ La psychologie sociale étudie l'individu (c'est une psychologie) dans des situations sociales réelles (elle est sociale), qui existent en dehors de lui, par lesquelles il est influencé, qu'il peut comprendre et influencer lui même »: Claude Revault d'Allonnes et Jacqueline Barus-Michel définissent ainsi dans le numéro du Bulletin de Psychologie (1980) qui lui est consacré, cette " science carrefour ». L'orientation clinique se spécifie par la prise en compte, au-delà des dimensions économiques et politiques, du registre du libidinal, à l'œuvre dans les relations sociales, qu'elles soient appréhendées aux niveaux interpersonnel, groupal, organisationnel ou institutionnel. Elle est aussi une pratique : le praticien se tient “ au pied du lit social », à la fois observateur et participant à la situation, à la fois objet, outil et acteur, “ obligé de tenir en main cette triple position ». Elle est une pratique d'accompagnement, à partir des demandes adressées, celles-ci renvoyant en dernière analyse, à une demande d'élucidation du sens des situations dans lesquels les acteurs sociaux se trouvent engagés.

“ L'objet de la psychologie sociale clinique est de comprendre les modes d'interférences et les processus d'engendrement du psychique et du social, soit qu'elle prenne appui sur les collectifs et unités sociales, soit qu'elle prenne appui sur les individus » (1996, p. 263).

Elle est donc à la fois une clinique du sujet (l'individu prétendant à faire sens) inscrit dans un champ social, inséré et traversé par une réalité sociale, individuellement ou au sein de collectifs, d'unités sociales ou culturelles plus ou moins larges; et une clinique des relations d'individus liés dans un collectif par des investissements et des pratiques, en rapport avec une réalité extérieure, cherchant à la transformer. Dans cette perspective, la problématique du social n'est pas seulement celle du lien, encore moins celle des seules déterminations sociales : elle est fondée sur la question de l'échange et de la coopération. "Le groupe, l'organisation, la société tissent des liens indispensables et insupportables pour faire ensemble, puisque l'homme ne peut vivre qu'en relation avec les autres pour transformer la réalité » (1996, p. 263).

\section{$\checkmark$ Complexité et multiréférentialité}

“ La psychosociale prétend analyser un objet complexe mais non moins réel, celui des individus confrontés à leurs semblables dans l'impérieuse nécessité de l'acte. Il 
y faut appréhender cette interaction du sujet, des autres et de la réalité. La réalité psychique y est intriquée à la réalité des collectifs institués et aucune ne peut se réduire à l'autre. (...) La complexité désigne l'interférence des différents registres, personnel, relationnel, fonctionnel, institutionnel, qui déterminent la dynamique des échanges et des positions » (1996, p. 265).

Le recours à la pluridisciplinarité s'impose alors : la psychanalyse, la phénoménologie, la psychologie sociale, la sociologie, l'ethnologie, l'histoire... et l'élaboration épistémologique portera essentiellement sur les modalités de leur utilisation conjointe. Dans son parcours épistémologique, Jacqueline Barus-Michel dialoguera avec Freud bien sûr, mais aussi avec le structuralisme de Lévi-Strauss, la sociopsychanalyse de Gérard Mendel, le courant de l'analyse institutionnelle de René Lourau et Georges Lapassade, la sociologie clinique de Vincent de Gaulejac...

\section{Vke sujet social}

“La psychosociale a affaire non seulement au sujet dans son expérience des situations sociales, mais aussi à ce sujet improbable que j'ai appelé le "sujet social”, celui qui prétend s'énoncer à la première personne du pluriel, "Nous", qu'il ne suffit pas de tenir pour la somme des individus qui s'y reconnaissent. C'est "un sujet pluriel" où chaque individu reste un sujet autonome tout en faisant partie du tout, reflet de l'ensemble » (2004, p. 23).

Le lien social ne peut être considéré comme une réduction ou une simple extension de l'intrapsychique. Le modèle psychofamilial de la sexualité infantile ne peut suffire à l'expliquer. La pulsion " sociale », fondamentalement ambivalente, qui se ne confond pas avec la pulsion sexuelle, définirait, avec elle, la nature propre de l'homme.

Le social est entendu ici comme rapports d'échanges et rapports de transformation de la réalité, c'est-à-dire comme praxis. Ce sont les relations (le lien et la rupture), les structures, les dynamiques, les systèmes symboliques, les discours et les actes qui en sont le produit et qui les reprennent. Jacqueline Barus-Michel souligne continûment que « le social ne vient pas qu'interdire, contraindre, réprimer, il instruit et contient, assurant une fonction d'étayage repérable dans les ruptures et les crises : ce qui se dérobe du social pour le sujet se saisit aussi comme manque d'étayage » (1996, p. 264).

Ce sujet social est donc individuel ou collectif (il prétend au “ je » ou au " nous »). Il peut être considéré isolément (individu) ou dans un lien solidaire (groupe, unité collective). Il est énonciateur d'un projet parmi et avec les autres, à la fois projet identitaire (expression, réalisation, reconnaissance) et projet de transformation de la réalité (coopération, production). 
Pour conclure cet impossible retour sur l'ensemble de son ouvre, nous retiendrons ici ce principe essentiel de la recherche clinique, tel que Jacqueline nous l'enseigné, comme à tous les étudiants qui ont eu le plaisir de bénéficier de sa passion de la transmission et de l'élaboration collective :

“Que cherche-t-on si ce n'est ce qui pose question à soi-même? Toute recherche comme celle d'Édipe pose les 3 questions fondamentales qui concernent le chercheur au premier chef: question d'origine, question de nature, question de destin (d'où viens-je? qui suis-je? où vais-je?).

Questions angoissantes, risquées, peut-être mortelles, si bien que le chercheur, dans un processus d'évitement, feint que son objet soit radicalement autre, jusqu'à dérisoire, jusqu'aux antipodes, jusqu'aux confins de l'univers... s'il est astrophysicien... Le chercheur en sciences humaines devrait être plus averti de sa propre inclusion dans la recherche. Mais le refoulement préside à la recherche en même temps que la curiosité passionnée qui en est le signe inversé. Le chercheur veut savoir sans se reconnaître. Son objet doit être différent, étranger, décidément ce qu’il n'est pas, ne peut pas être. Et même, cette différence est destinée à le protéger, comme si à être médecin on se garantissait de n'être jamais malade. Mais il n'atteindra de la différence que si il s'y reconnaît d'abord. On peut se demander si toute rigueur de la recherche ne se garantit d'abord dans un examen du refoulement, à savoir du chemin qui a conduit le chercheur à énoncer son désir dans les termes de ses hypothèses et sous les espèces de son objet " ${ }^{[4]}(1986$, p. 803).

Jacqueline nous a quittés. Elle nous laisse pour poursuivre avec elle l'aventure, “ à la recherche d'un supposé savoir " ${ }^{[5]}$ dont la preuve passe par l'épreuve, nombres d'ouvrages et articles, qui peuvent nous guider, nous éclairer et faire revivre cette Grande Dame.

Dominique Lhuillier CRTD-Cnam

- L'énergie du paradoxe. Paris : Desclée de Brouwer, 2013.

- Désir, passion, érotisme. L'expérience de la jouissance. Toulouse : Érès, Collection Sociologie clinique, 2009.

- Le politique entre les pulsions et la loi. Toulouse : Érès, Collection Sociologie clinique, 2007.

- Souffrance, sens et croyance. L'effet thérapeutique. Toulouse : Érès, Collection Sociologie clinique, 2004 .

- Pouvoir : mythe et réalité. Paris : Klincksieck, coll. Rencontres dialectiques, 1991.

[4] Barus-Michel J. (1986). Le chercheur, premier objet de la recherche, Bulletin de Psychologie, tome XXXIX, $\mathrm{n}^{\circ} 377$, 801-804.

[5] Barus-Michel J. (2009). À la recherche d'un supposé savoir, in Giust-Desprairies et de Gaulejac V. (s/dir). La subjectivité à l'épreuve du social, Paris : I'Harmattan, 257-270. 


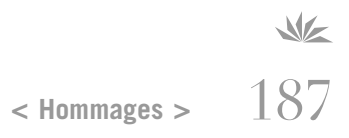

- Le sujet social. Étude de psychologie sociale clinique. Paris : Dunod, coll. Organisation et Sciences humaines, 1987.

- Avec E. Enriquez et A. Lévy, Vocabulaire de Psychosociologie. Références et positions. Toulouse : Érès, 2002, 11 éd., 2013.

- Avec F. Giust-Desprairies et L. Ridel, Crises. Approche psychosociale clinique. Paris : Desclée de Brouwer, coll. Reconnaissances, 1996. 